\title{
Kosmotropes and chaotropes: modelling preferential exclusion, binding and aggregate stability
}

\author{
Susanne Moelbert \\ Institut de théorie des phénomènes physiques, Ecole polytechnique fédérale de Lausanne, CH-1015 Lausanne, Switzerland \\ B. Normand \\ Département de Physique, Université de Fribourg, CH-1700 Fribourg, Switzerland \\ Paolo De Los Rios \\ Institut de théorie des phénomènes physiques, Ecole polytechnique fédérale de Lausanne, CH-1015 Lausanne, Switzerland and \\ INFM UdR - Politecnico, Corso Duca degli Abruzzi 24, 10129 Torino, Italy.
}

(Dated: March 19, 2018)

\begin{abstract}
Kosmotropic cosolvents added to an aqueous solution promote the aggregation of hydrophobic solute particles, while chaotropic cosolvents act to destabilise such aggregates. We discuss the mechanism for these phenomena within an adapted version of the two-state Muller-Lee-Graziano model for water, which provides a complete description of the ternary water/cosolvent/solute system for small solute particles. This model contains the dominant effect of a kosmotropic substance, which is to enhance the formation of water structure. The consequent preferential exclusion both of cosolvent molecules from the solvation shell of hydrophobic particles and of these particles from the solution leads to a stabilisation of aggregates. By contrast, chaotropic substances disrupt the formation of water structure, are themselves preferentially excluded from the solution, and thereby contribute to solvation of hydrophobic particles. We use Monte Carlo simulations to demonstrate at the molecular level the preferential exclusion or binding of cosolvent molecules in the solvation shell of hydrophobic particles, and the consequent enhancement or suppression of aggregate formation. We illustrate the influence of structure-changing cosolvents on effective hydrophobic interactions by modelling qualitatively the kosmotropic effect of sodium chloride and the chaotropic effect of urea.
\end{abstract}

PACS numbers: 64.75.+g, 61.20.-p, 87.10.+e, 64.70.Ja

\section{INTRODUCTION}

Most processes in living organisms are adjusted to function in a rather limited range of physiological conditions, and important deviations, such as high concentrations of dangerous substances, are generally expected to preclude life. Nevertheless, many living systems survive stresses of this kind and exist in hostile environments. One common adaptation strategy is to modify the properties of the solvent, which is usually water, in such a way as to exclude the undesirable solutes from solution [1]. Water is modified by relatively high concentrations of stabilising solutes (cosolvents), which remain compatible with the metabolism of the cell even at very high concentrations (therefore they are also referred to as 'compatible osmolytes') [2, 3]. These cosolvents neutralise dangerous solutes by decreasing their solubility and enhancing the formation of their aggregates. Such cosolvents are known as promoters of the water structure and are therefore referred to as kosmotropes ('kosmo-trope' = order maker). Their stabilising function for proteins and aggregates of hydrophobic solute particles, and their importance for the osmotic balance in cells, has generated a growing interest in the physical origin of the kosmotropic effect. Many recent investigations have focused on the ability of kosmotropic cosolvents to influence solute solubility in aqueous media [4, 5, [6, 7, 8, 9].

Of equal interest and importance is the ability of chaotropic cosolvents to increase the solubility of nonpolar solute particles in aqueous solutions 10, 11, 12, 13, 14, 15]. For certain systems the solubility may be enhanced by several orders of magnitude [16, 17, 18], leading to a complete destabilisation of solute aggregates and, in the case of protein solutions, to a complete denaturation and loss of function. A significant number of substances display this property, notably (under most conditions and for the majority of solute species) urea, which is frequently used in highly concentrated (c. 0.5-10 M) solutions as a protein denaturant. Chaotropic cosolvents ('chao-tropic' $=$ order-breaking) are less polar than water and thus break hydrogen bonds between water molecules, suppressing water structure formation [5, 19, 20, 21]. However, the exact physical mechanism for the changes in water structure, and for the consequent destabilising action of chaotropic cosolvents, is at present not fully understood.

Because the origin of the kosmotropic and chaotropic effects appears to lie primarily in their influence on the solvent, rather than in direct interactions between cosolvent and solute [22, 23, 24], a microscopic description must begin with the unique properties of the aqueous medium. Water molecules have the ability to form strong, intermolecular hydrogen bonds, and pure, liquid water may form extended hydrogen-bonded networks, becoming highly ordered (Fig. 11). Although the insertion of a hydrophobic molecule leads to a destruction of lo- 


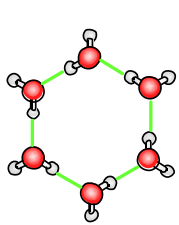

ice structure

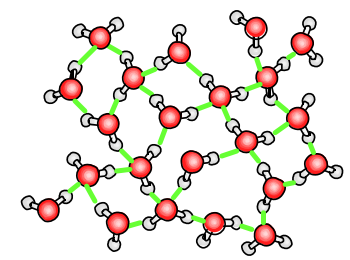

ordered liquid water

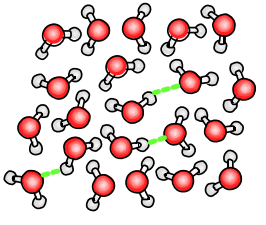

disordered liquid water
FIG. 1: Schematic illustration of water structure as a consequence of the formation of more or less extended networks of hydrogen bonds.

cal hydrogen-bond structure, at low temperatures water molecules are found to rearrange in a cage-like configuration around small solute particles and around highcurvature regions of larger ones. The orientation imposed on the water molecules at the interface with the particle results in the cage hydrogen bonds being slightly stronger than before, and causing a net reduction of energy during the insertion process [25, 26, 27, 28, 29]. However, at higher temperatures the free energy of the system is decreased by reducing the local restructuring of water, thus increasing the entropy of the solvent molecules, which drives the aggregation of hydrophobic particles through a minimisation of their total surface exposed to water. This competition between enthalpic and entropic effects in the solvent is fundamental to the phenomenology of liquid-liquid demixing processes. The effective hydrophobic interaction between small, non-polar solute particles is thus thought to be primarily solvent-induced, i.e. to be a consequence of changes in the ordering of water molecules rather than being controlled by direct watersolute interactions [30, 31, 32, 33, 34]. In this description, the primary contribution to the action of structurechanging cosolvents, which are generally highly soluble and uncharged in physiological conditions, then depends on their ability to alter this local ordering of liquid water.

Kosmotropic cosolvents, such as sucrose and betaine, are more polar than water and act to enhance its structure due to their ability to form hydrogen bonds [5]. For the same reason they interact with water molecules rather than with non-polar solute particles, which leads to an effective preferential exclusion from the solvation shell of hydrophobic molecules [1, 6, 24], and thus to a stronger net repulsion between solute and solvent. In the presence of kosmotropic cosolvents, structural arrangement of the water-cosolvent mixture is enthalpically favourable compared to a cage-like organisation around hydrophobic solute particles. Solute molecules are thus pushed together to minimise their total exposed surface, which results in an enhancement of hydrophobic aggregation. The same process leads to a stabilisation of native protein configurations, in spite of the fact that kosmotropic substances have no net charge and do not interact directly with the proteins [5, 7, 8].

Chaotropic cosolvents, such as urea in most ternary
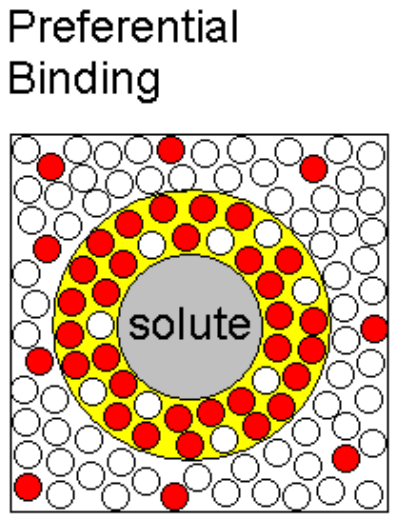

water Preferential
Exclusion

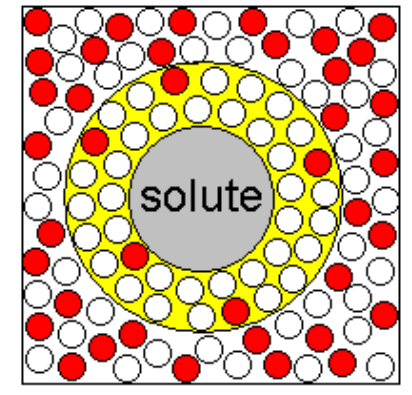

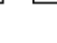

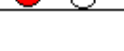

\section{cosolvent}

FIG. 2: Schematic representation of preferential phenomena in a mixture of water and hydrophobic solute particles in the presence of chaotropic (left) and kosmotropic (right) cosolvents (after Ref. [24]).

systems, are less polar than water, so that their presence in solution leads to an energetically unfavourable disruption of water structure. Such cosolvents are therefore excluded from bulk water, an effect known as "preferential binding" to the solute particles [11, 24], although it relies less on any direct binding of cosolvent to solute (which would enhance the effect) than on the fact that the cosolvent molecules are pushed from the solvent into the shell regions of the solute. Preferential binding and exclusion of cosolvent molecules are depicted in Fig. 2. In the former case, the smaller number of water molecules in contact with the surface of non-polar solute particles leads to a weaker effective interaction between solute and solvent, such that a larger mutual interface becomes favourable. The addition of chaotropic cosolvents to aqueous solutions therefore results in an increase in solvent-accessible surface area which destabilises hydrophobic aggregates, micelles and native protein structures [10, 11, 24, 35].

The essential phenomenology of the kosmotropic and chaotropic effects may be explained within a solventbased model founded on the concept of two physically distinct types of solvent state, namely ordered and disordered water [4, 5] (Fig. 11). In Sec. II we review this model, described in detail in Ref. [36], and focus on the adaptations which represent the structurechanging effects of the cosolvents. Sec. III presents the results of Monte Carlo simulations for kosmotropic substances, which illustrate their stabilising effect on hydrophobic aggregation, the decrease in solubility of non-polar molecules in water-cosolvent mixtures and the underlying preferential exclusion of cosolvent molecules from the solvation shell of hydrophobic particles. We consider the inverse phenomena of the chaotropic effect in Sec. IV In Sec. D we illustrate these results by comparison with available experimental data for a kosmotropic 
and a chaotropic cosolvent, and discuss the extent of the symmetry between the two effects in the context of the model description for weakly or strongly active agents. Sec. VI contains a summary and conclusions.

\section{MODEL}

\section{A. Hydrophobic-Polar Model}

The driving force in the process of solvation and aggregation is the effective hydrophobic interaction between polar water and the non-polar solute [23, 30]. As noted in Sec. I, the origin of this interaction is the rearrangement of water around the solute particle. For solute surfaces of sufficiently high curvature to permit (partial) cage formation, this process decreases the enthalpy due to reinforced hydrogen bonds between water molecules in the solvation shell of the hydrophobic particle in comparison to those in bulk water, but reduces simultaneously the number of degenerate states of the solvent. These physical features are described by the model of Muller, Lee and Graziano (MLG), where the energy levels and respective degeneracies of water molecules are determined by the local water structure [37, 38].

Because solute particles are relatively large compared with single water molecules, we use an adapted version of the MLG model in which each site contains a group of water molecules. The distinction between solvent molecules and non-polar solute particles lies in their ability to form hydrogen bonds. The continuous range of interaction energies within a partially hydrogen-bonded water cluster may be simplified to two discrete states of predominantly intact or broken hydrogen bonds [39]. The water sites in the coarse-grained model may then be characterised by two states, where an "ordered" site represents a water cluster with mostly intact hydrogen bonds, while a "disordered" site contains relatively fewer intact hydrogen bonds (Fig. 11). The use of a bimodal distribution in the adapted model is not an approximation, but a natural consequence of the two-state nature of the pure MLG model [36]. In the presence of a non-polar solute a further distinction is required, between "bulk" water sites undisturbed by the solute particles and "shell" sites in their vicinity whose hydrogen bonding is altered. As explained in more detail below, this adaptation of the MLG model contains the essential features required to encapsulate the enthalpy/entropy balance which is the basis for the primary phenomena of hydrophobic aggregation in two-component water/solute mixtures. The model has been used to reproduce the appearance of upper (UCST) and lower (LCST) critical solution temperatures and a closed-loop coexistence regime [40], demonstrating that the origin of the hydrophobic interaction lies in the alteration of water structure.

The adapted MLG model has been extended to provide a minimal model for the study of chaotropic phenomena in ternary water/solute/cosolvent systems [36]. It was

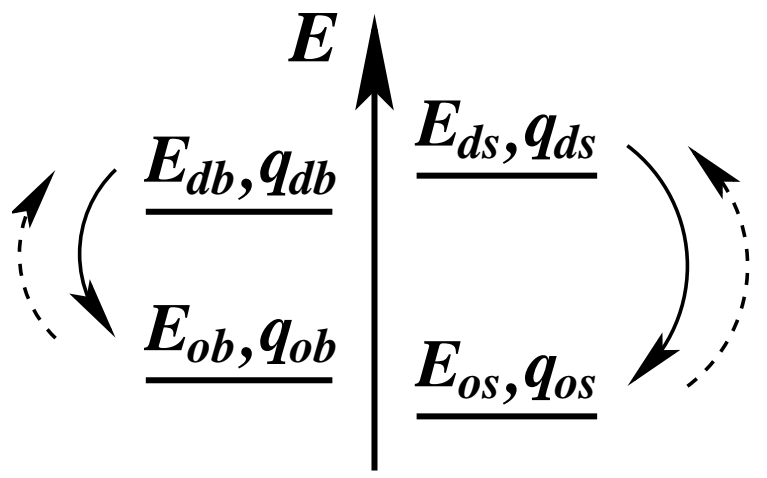

FIG. 3: Energy levels of a water site in the bimodal MLG model. The energy levels of shell water are different from bulk water due to breaking and rearrangement of hydrogen bonds in the proximity of a solute particle. The solid arrows represent the effect of a kosmotropic cosolvent in creating more ordered states, and the dashed arrows the opposite effect of chaotropic species.

shown that this framework yields a successful description of preferential binding, and of the resulting destabilisation of aggregates of solute particles as a consequence of the role of chaotropic cosolvents in reducing the formation of water structure. We begin with a brief review of the model to summarise its physical basis and to explain the modifications required for the inclusion of cosolvent effects.

The microscopic origin of the energy and degeneracy parameters for the four different types of water site (ordered/disordered and shell/bulk) is discussed in Refs. 36, 41, 42], in conjunction with experimental and theoretical justification for the considerations involved. Here we provide only a qualitative explanation of their relative sizes, which ensure the competition of enthalpic and entropic contributions underlying the fact that the model describes a closed-loop aggregation region bounded by upper and lower critical temperatures and densities [40]. Considering first the energies of water sites (groups of molecules), the strongest hydrogen bonding arises not in ordered bulk water, but in the shell sites of hydrophobic particles due to the cage formation described in Sec. I (which can be considered as a result of the orientational effect of a hydrophobic surface). Both types of ordered water cluster are enthalpically much more favourable than disordered groups of molecules, for which the presence of a non-polar particle serves only to reduce hydrogen bonding still further, making the disordered shell energy the highest of all. As regards the site degeneracies, which account for the entropy of the system, cage formation permits very few different molecular configurations, and has a low degeneracy. Water molecules in ordered clusters have significantly reduced rotational degrees of freedom compared to disordered ones, with the result that the degeneracy of the latter is considerably higher. Finally, the degeneracy of a disordered shell site is still higher than that of a bulk 
site because the relative reduction of hydrogen bonding due to the non-polar surface admits a higher amount of rotational freedom.

Figure 3 summarises the energy levels of a water site, which are arranged in the sequence $E_{d s}>E_{d b}>E_{o b}>$ $E_{o s}$. Their respective degeneracies are $q_{d s}>q_{d b}>q_{o b}>$ $q_{o s}$, where the states are denoted $d s=$ disordered shell, $d b=$ disordered bulk, $o b=$ ordered bulk and $o s=$ ordered shell (cage conformation). We stress that these sequences are neither an assuption of the model nor may they be altered if the model is to represent a system exhibiting the closed-loop form of the coexistence curve for aggregation: only these specific sequences reproduce appropriately the competition of enthalpic and entropic contributions to the free energy. From the microscopic considerations of the previous paragraph these sequences are entirely plausible, and they have been confirmed by a range of experimental measurements; the exact values of the parameters are not important for the qualitative properties of the system while the order of energies and degeneracies is maintained. We return in Sec. $\mathrm{V}$ to the issue of departure from these sequences.

In the calculations to follow we have used the energy values $E_{d s}=1.8, E_{d b}=1.0, E_{o b}=-1.0$ and $E_{o s}=-2.0$, which are thought to be suitably representative for aqueous solutions, and which have been successful in describing a number of different types of solution 40, 41, 43]. Their corresponding degeneracies, normalised to a non-degenerate ordered shell conformation, are taken as $q_{d s}=49, q_{d b}=40, q_{o b}=10$ and $q_{o s}=1$ [41]. We remind the reader that these energy and degeneracy parameters are independent of temperature and solute density. Their relative values have been found to be appropriate for reproducing the primary qualitative features of hydrophobic interactions 40, 41], swelling of biopolymers 43], protein denaturation [44, 45], and micelle formation [46]. The essential behaviour obtained within this model is indeed found to be rather insensitive to the precise parameter values, which may, however, be refined by comparison with experimental measurements to yield semi-quantitative agreement for different solutions [40, 41, 43]. The energy scale is correlated directly with a temperature scale, which we define as $k_{B} T \equiv \beta^{-1}$.

We conclude this subsection by qualifying the range of validity of the adapted MLG model, including what is meant above by "small" solute particles. The model is not appropriate for solute species smaller than a group of water molecules with intercluster hydrogen-bonding, which necessitates a linear cluster (and solute) size of at least 2 water molecules. The model will also fail to capture the physics of large, uniform systems where the linear cluster size exceeds perhaps 10 water molecules, because in this case the majority of the water molecules in a "shell site" would correspond effectively to bulk water. Most molecular-scale treatments of water structure in the vicinity of non-polar solute particles assume a spherical and atomically flat solute surface. These analyses [47, 48, 49] indicate that "clathrate" models for the (partial) formation of hydrogen-bonded cages become inappropriate for larger curvatures, on the order of $1 \mathrm{~nm}$, where a crossover occurs to "depletion" or "dangling-bond" models in which hydrogen-bond formation is frustrated [50]. While the adapted MLG model becomes inapplicable for such particles at the point where a clathrate description breaks down, we note [46] that most solute species, and in particular proteins, are atomically rough, in that they composed of chains and side-groups with high local curvatures on the order of $0.3-0.5 \mathrm{~nm}$. As a consequence the model may remain valid for solute particles of considerably greater total dimension. For the purposes of the present analysis, we note that a significant quantity of the available data concerning cosolvent effects has been obtained for protein solutions, and that the majority of this data is consistent with our basic picture of the dominance of water structure effects in dictating solute solubility. However, while most proteins may indeed fall in the class of systems well described by a clathrate picture, we stress that the adapted MLG model cannot be expected to provide a complete description for large solute molecules containing many amino-acids of different local hydrophobicity and chemical interactions.

\section{B. Cosolvent Addition}

In comparison with the hydrophobic solute particles, cosolvent molecules are generally small and polar, and are therefore included directly in water sites by changing the number of states of these sites. A site containing water molecules and a cosolvent particle is referred to as a cosolvent site. Kosmotropic cosolvents, being more polar than water, increase the number of intact hydrogen bonds at a site [19]. In the bimodal MLG framework their addition to weakly hydrogen-bonded, disordered clusters may be considered to create ordered clusters with additional intact hydrogen bonds. The creation of ordered states from disordered ones in the presence of a kosmotropic cosolvent increases the degeneracy of the former at the expense of the latter. This feature is incorporated by raising the number of possible ordered states compared to the number of disordered states (solid arrows in Fig. 3),

$$
\begin{aligned}
q_{o b, k}=q_{o b}+\eta_{b}, & q_{d b, k}=q_{d b}-\eta_{b}, \\
q_{o s, k}=q_{o s}+\eta_{s}, & q_{d s, k}=q_{d s}-\eta_{s},
\end{aligned}
$$

where $k$ denotes the states of water clusters containing kosmotropic cosolvent molecules, and the total number of states is kept constant.

The effect of chaotropic cosolvents on the state degeneracies is opposite to that of kosmotropic substances, and may be represented by inverting the signs in eq. 1] Chaotropic cosolvents are less strongly polar than water, acting in an aqueous solution of hydrophobic particles to reduce the extent of hydrogen bonding between water molecules in both shell and bulk sites [23, 51]. Within the adapted MLG framework, this effect is re- 
produced by the creation of disordered states, with additional broken hydrogen bonds and higher enthalpy, from the more strongly bonded ordered clusters (dashed arrows in Fig. [3), whence

$$
\begin{aligned}
q_{o b, c}=q_{o b}-\eta_{b}, & q_{d b, c}=q_{d b}+\eta_{b}, \\
q_{o s, c}=q_{o s}-\eta_{s}, & q_{d s, c}=q_{d s}+\eta_{s} .
\end{aligned}
$$

We stress three important qualitative points. First, the cosolvent affects only the number of intact hydrogen bonds, but not their strength [23], as a result of which, also in the bimodal distribution of the coarse-grained model, the energies of the states remain unchanged. Cosolvent effects are reproduced only by the changes they cause in the relative numbers of each type of site [eqs. (1) and (2)]. Secondly, cosolvent effects on the relative degeneracy parameters are significantly stronger in the bulk than in the shell, because for obvious geometrical reasons related to the orientation of water molecules around a non-polar solute particle 36, 41], many more hydrogenbonded configurations are possible in the bulk. Finally, as suggested in Sec. I, our general framework does not include the possibility of cosolvent-solute interactions, which have been found to be important in certain ternary systems, specifically those containing urea [52, 53].

The illustrative calculations in Secs. III and IV are performed with $\eta_{b}=9.0$ and $\eta_{s}=0.1$ in eqs. (11) and (2). In the analysis of Ref. [36] these values were found to provide a good account of the qualitative physics of urea as a chaotropic cosolvent; in Sec. V we will return to a more quantitative discussion of the role of these parameters. By using the same values for a hypothetical kosmotropic cosolvent we will demonstrate that a symmetry of model degeneracy parameters does not extend to a quantitative, or even qualitative, symmetry in all of the relevant physical phenomena caused by structure-changing cosolvents. We comment briefly that the fractional value of $\eta_{s}$ arises only from the normalisation convention $q_{o s}=1$.

\section{Mathematical Formulation}

To formulate a description of the ternary solution within the framework of statistical mechanics, we begin by associating with each of the $N$ sites of the system, labelled $i$, a variable $n_{i}$, which takes the values $n_{i}=1$ if the site contains either pure water, $n_{i}=0$ if the site contains a hydrophobic particle, or $n_{i}=-1$ if the site contains a water cluster including one or more cosolvent molecules. This system is described by the Hamiltonian

$$
\begin{aligned}
H & {\left[\left\{n_{i}\right\},\left\{\sigma_{i}\right\}\right]=\sum_{i=1}^{N} \frac{n_{i}\left(n_{i}+1\right)}{2}\left[\left(E_{o b} \tilde{\delta}_{i, \sigma_{o b}}+E_{d b} \tilde{\delta}_{i, \sigma_{d b}}\right) \lambda_{i}\right.} \\
& \left.+\left(E_{o s} \tilde{\delta}_{i, \sigma_{o s}}+E_{d s} \tilde{\delta}_{i, \sigma_{d s}}\right)\left(1-\lambda_{i}\right)\right] \\
& +\sum_{i=1}^{N} \frac{n_{i}\left(n_{i}-1\right)}{2}\left[\left(E_{o b} \tilde{\delta}_{i, \sigma_{o b, a}}+E_{d b} \tilde{\delta}_{i, \sigma_{d b, a}}\right) \lambda_{i}\right. \\
& \left.+\left(E_{o s} \tilde{\delta}_{i, \sigma_{o s, a}}+E_{d s} \tilde{\delta}_{i, \sigma_{d s, a}}\right)\left(1-\lambda_{i}\right)\right],
\end{aligned}
$$

where $a=k, c$ denotes sites containing water and a kosmotropic or chaotropic cosolvent molecule. The site variable $\lambda_{i}$ is defined as the product of the nearest neighbours, $\lambda_{i}=\prod_{\langle i, j\rangle} n_{j}^{2}$, and takes the value 1 if site $i$ is completely surrounded by water and cosolvent or 0 otherwise. The first sum defines the energy of pure water sites and the second the energy of cosolvent sites. Because a water site $i$ may be in one of $q$ different states, $\tilde{\delta}_{i, \sigma_{o s}}$ is 1 if site $i$ is occupied by water in one of the $q_{o s}$ ordered shell states and 0 otherwise, and $\tilde{\delta}_{i, \sigma_{d s}}$ is 1 if it is occupied by pure water in one of the $q_{d s}$ disordered shell states and 0 otherwise. Analogous considerations apply for the bulk states and for the states of cosolvent sites. A detailed description of the mathematical structure of the model is presented in Refs. [40] and [36].

The canonical partition function of the ternary $N$-site system, obtained from the sum over the state configurations $\left\{\sigma_{i}\right\}$, is

$$
\begin{aligned}
Z_{N}=\sum_{\left\{n_{i}\right\}} \prod_{i} & Z_{s}^{\frac{n_{i}\left(n_{i}+1\right)}{2}\left(1-\lambda_{i}\right)} Z_{b}^{\frac{n_{i}\left(n_{i}+1\right)}{2} \lambda_{i}} \\
& \times Z_{s, a}^{\frac{n_{i}\left(n_{i}-1\right)}{2}\left(1-\lambda_{i}\right)} Z_{b, a}^{\frac{n_{i}\left(n_{i}-1\right)}{2}} \lambda_{i}
\end{aligned}
$$

where $Z_{\sigma}=q_{o \sigma} e^{-\beta E_{o \sigma}}+q_{d \sigma} e^{-\beta E_{d \sigma}}$ for the shell $(\sigma=$ $s)$ and bulk $(\sigma=b)$ states both of pure-water and of cosolvent sites $(\sigma=s, a$ and $\sigma=b, a)$.

When the number of particles may vary, a chemical potential is associated with the energy of particle addition or removal. The grand canonical partition function of the system for variable particle number becomes

$$
\Xi=\sum_{N} e^{\beta \mu N_{w}+\beta(\mu+\Delta \mu) N_{a}} Z_{N}=\sum_{\left\{n_{i}\right\}} e^{-\beta H_{\mathrm{eff}}^{\mathrm{gc}}\left[\left\{n_{i}\right\}\right]} .
$$

$N_{w}$ denotes the number of pure water sites, $N_{a}$ the number of cosolvent sites and $N_{p}$ the number of solute particle sites, the total number being $N=N_{w}+N_{a}+N_{p}$. The variable $\mu$ represents the chemical potential associated with the addition of a water site to the system and $\Delta \mu$ the chemical potential for the insertion of a cosolvent molecule at a water site. From the role of the two cosolvent types in enhancing or disrupting water structure, at constant solute particle density $\Delta \mu$ is expected to be negative for kosmotropic agents and positive for chaotropic ones.

\section{Monte Carlo Simulations}

We describe only briefly the methods by which the model may be analyzed; full technical details may be found in Ref. [40]. Because our primary interest is in the local solute and cosolvent density variations which demonstrate aggregation and preferential phenomena, we focus in particular on Monte Carlo simulations at the level of individual solute molecules. Classical Monte Carlo simulations allow the efficient calculation of thermal averages in many-particle systems with statistical 
fluctuations [40, such as that represented by eq. (5). We work on a cubic lattice of $30 \times 30 \times 30$ sites $(N=27000)$, with random initial particle distributions and with periodic boundary conditions to eliminate edge effects (although clearly we cannot eliminate finite-size effects). Each site is occupied by either a solute particle, pure water or a water-cosolvent mixture. The results are unaffected by changes in lattice size. We have implemented a Metropolis algorithm for sampling of the configuration space. After a sufficiently large number $(100000)$ of relaxation steps, the system achieves thermal equilibrium and averages are taken over a further 500000 measurements to estimate thermodynamic quantities. Coexistence lines in the $\mu-T$ phase diagram are obtained from the transitions determined by increasing the temperature at fixed chemical potential $\mu$ (grand canonical sampling), which results in a sudden density jump at the transition temperature. The solute particle densities $\rho_{p}$ corresponding to these jumps yield closed-loop coexistence curves in the $\rho_{p}-T$ phase diagram. Despite the rather crude approximation to a continuum system offered by the use of a cubic lattice, we have found quantitative agreement with other theoretical approaches, which is why we focus primarily on Monte Carlo simulations here. In Sec. V we will also demonstrate remarkable qualitative, and in some cases semi-quantitative, agreement with experiment, suggesting that the adapted MLG framework may indeed form a suitable basis for more sophisticated modelling.

For the macroscopic properties of the system, such as aggregation and phase coexistence, the results of the Monte Carlo simulations may be interpreted with the aid of a mean-field analysis. If the densities at each site are approximated by their average values in the solution, $\rho_{p}$ for hydrophobic particles, $\rho_{w}$ for pure water and $\rho_{a}$ for cosolvent, where $\rho_{p}+\rho_{w}+\rho_{a}=1$, the mean value $\left\langle n_{i}\right\rangle$ at site $i$ is given by $\left\langle n_{i}\right\rangle=\sum_{\sigma} n_{i, \sigma} \rho_{\sigma}=\rho_{w}-\rho_{a}$ and analogously $\left\langle n_{i}^{2}\right\rangle=\sum_{\sigma} n_{i, \sigma}^{2} \rho_{\sigma}=\rho_{w}+\rho_{a}$. The equilibrium densities are obtained by minimisation of the grand canonical mean-field free energy per site,

$$
\begin{aligned}
f= & \left(\mu-\beta^{-1} \ln Z_{s}\right) \rho_{w}+\left(\mu+\Delta \mu-\beta^{-1} \ln Z_{s, a}\right) \rho_{a} \\
& +\beta^{-1}\left(\ln Z_{s}-\ln Z_{b}\right) \rho_{w}\left(\rho_{a}+\rho_{w}\right)^{z} \\
& +\beta^{-1}\left(\ln Z_{s, a}-\ln Z_{b, a}\right) \rho_{a}\left(\rho_{a}+\rho_{w}\right)^{z} \\
& +\beta^{-1}\left(\rho_{w} \ln \rho_{w}+\rho_{a} \ln \rho_{a}+\rho_{p} \ln \rho_{p}\right),
\end{aligned}
$$

where $z$ denotes the number of nearest neighbours of each site.

\section{KOSMOTROPIC EFFECT}

We begin our analysis of the kosmotropic effect by discussing the mean-field $\rho_{p}$-T phase diagram for different

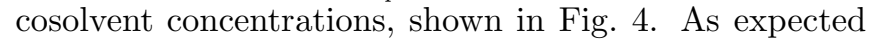
from Sec. IIA the system exhibits a closed-loop coexistence curve, forming a homogeneous particle-solventcosolvent mixture below a LCST and above an UCST for all concentrations, whereas between these temperatures,

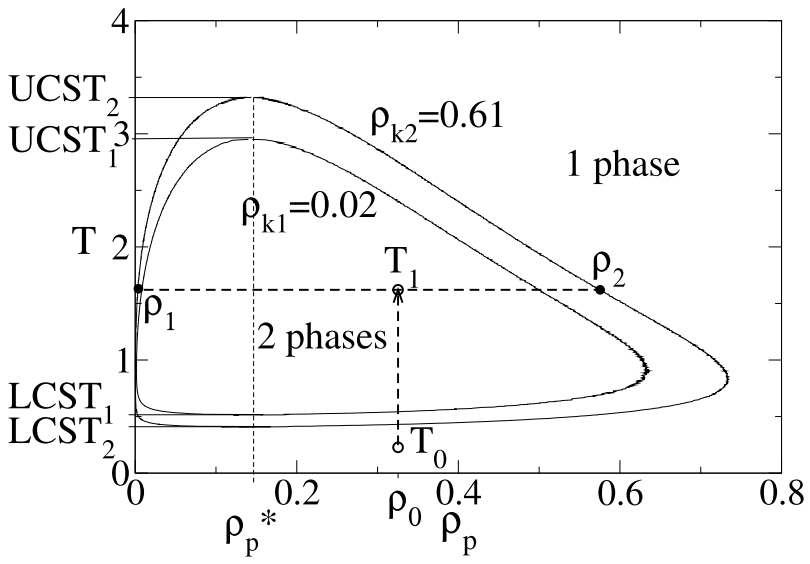

FIG. 4: Closed-loop coexistence curves for a ternary system of water, kosmotropic cosolvent and hydrophobic particles for two different cosolvent densities $\rho_{k}$, obtained by mean-field calculation with degeneracy parameters $\eta_{b}=9.0$ and $\eta_{s}=$ 0.1 in eq. (1). On the outside of each curve the solution is homogeneous, whereas on the inside it separates into two phases. The dashed arrow represents the heating process of a system with particle density $\rho_{0}$ and cosolvent density $\rho_{k 2}=$ 0.61 from temperature $T_{0}$ in the homogeneous region to $T_{1}$ in the two-phase region. At $T_{1}$ the system is separated into two phases of different densities $\rho_{1}$ (nearly pure water-cosolvent mixture) and $\rho_{2}$ (hydrophobic aggregates).

and for intermediate particle concentrations, a phase separation is found into a pure solvent phase (meaning a water-cosolvent mixture) and an aggregated phase with fixed solute density.

The hydrophobic repulsion between water and nonpolar solute particles, developed as a result of the formation of water structure, increases in the presence of kosmotropic cosolvents (this result may be shown by rewriting the partition function (eq. 5) in terms of the particle sites [36]). As shown in Fig. [4 the temperature and density ranges of the aggregation region indeed rise with increasing cosolvent concentration, illustrating the stabilising effect of kosmotropic agents. Further, the particle density of the aggregate phase in the two-phase region increases when adding cosolvent, demonstrating in addition the strengthening of effective hydrophobic interactions between solute particles due to the growing water-solute repulsion. This is illustrated by the process of heating a system at constant density: the dashed arrow in Fig. 4 represents a solution with particle density $\rho_{0}$, which is heated from temperature $T_{0}$ in the homogeneous region to a temperature between the LCST and the UCST. In the heterogeneous region $\left(T_{1}\right)$, the solution separates into two phases of densities $\rho_{1}$ (almost pure solvent) and $\rho_{2}$ (hydrophobic aggregates) under the constraint $\rho_{0}\left(V_{1}+V_{2}\right)=\rho_{1} V_{1}+\rho_{2} V_{2}$, where $V_{i}$ is the volume occupied by phase $i$. An increase in cosolvent density leads to a higher density $\rho_{2}$ of hydrophobic ag- 


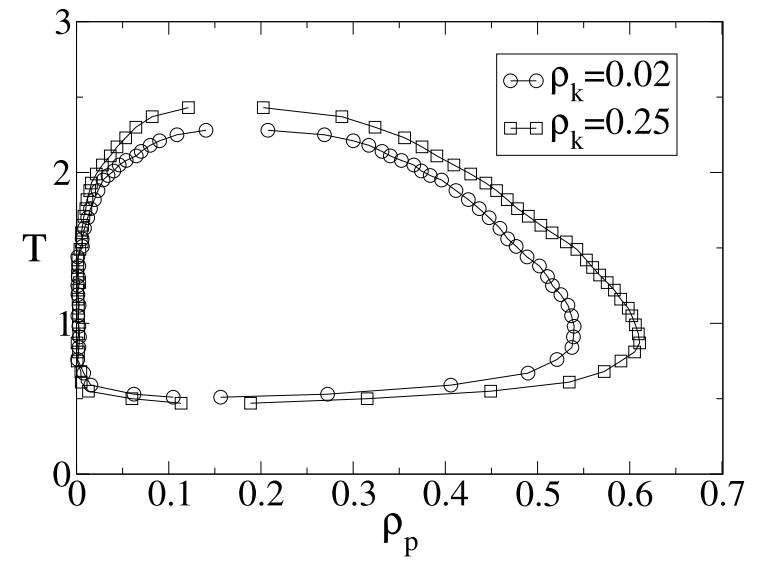

FIG. 5: Closed-loop coexistence curves obtained by Monte Carlo simulations for an aqueous solution of hydrophobic particles with the same parameters as in Fig. 4

gregates and a lower value $\rho_{1}$, which may be interpreted as a strengthening of the hydrophobic interactions generated between solute particles.

However, the mean-field approximation neglects fluctuations in the density, and consequently is unable to detect intrinsically local phenomena such as preferential exclusion of cosolvent particles from the solvation shell of a hydrophobic particle (Fig. 22). Because the kosmotropic effect is strongly dependent upon local enhancement of water structure, a full analysis is required of the spatial density fluctuations in the system. We thus turn to the results of Monte Carlo simulations, which at the macroscopic level show the same enhancement of hydrophobic aggregation (Fig. 5) as indicated in the mean-field analysis. Below the LCST and above the UCST, one homogeneous mixture is found where the hydrophobic particles are dissolved, while between these temperatures they form aggregates of a given density. The LCST and the critical aggregate densities determined by the mean-field calculation do not differ strongly from the numerical results, but the UCST lies at a temperature higher than that determined by Monte Carlo simulations. This is to be expected because mean-field calculations neglect fluctuation effects, generally overestimating both transition temperatures. The agreement of the mean-field result for small $\rho_{k}$ with the simulated value for the LCST suggests a predominance of local effects at low temperatures, and that density fluctuations between sites are small. We note, however, that a cosolvent concentration $\rho_{k}=0.61$ is required in the mean-field approximation to produce an expansion of the aggregated phase similar to that observed in the Monte Carlo results for $\rho_{k}=0.25$, demonstrating the growing importance of fluctuation effects at higher cosolvent concentrations.

The $\mu-T$ phase diagram of the ternary system obtained by Monte Carlo simulations is presented in Fig. [6] Lines

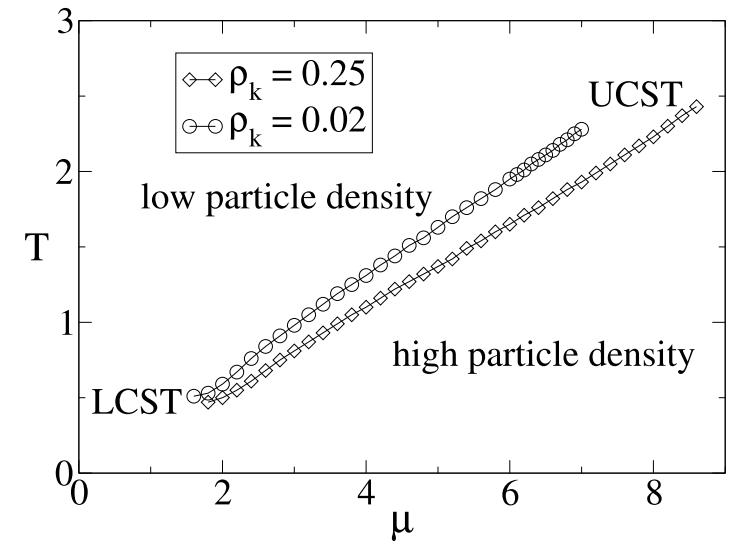

FIG. 6: $\mu-T$ phase diagram of hydrophobic solute in an aqueous cosolvent mixture for two different kosmotropic cosolvent concentrations $\rho_{k}$, obtained by Monte Carlo simulations. The endpoints of the finite transition lines correspond to the UCST and LCST.

of finite length terminated by the UCST and LCST demarcate the aggregation phase transition. An increase in cosolvent density leads to a larger separation of UCST and LCST as a direct result of the expansion of the aggregation regime (Fig. 5). The transition line is shifted to higher values of $\mu$, indicating an increased resistance of the system to addition of water at constant volume in the presence of cosolvent. This is a consequence of the fact that the resistance to addition of hydrophobic particles depends on both $\mu$ and $\Delta \mu$ : with increasing cosolvent concentration, $\Delta \mu$ decreases (i.e. becomes more negative) and hence $\mu$ must increase for the critical particle density to remain constant.

By inspection of the energy levels, and of the alteration in their relative degeneracies caused by a kosmotropic cosolvent (Fig. 3), it is energetically favourable to maximise the cosolvent concentration in bulk water with respect to shell water. Thus one expects a preferential exclusion, also known as preferential hydration, as represented schematically in the right panel of Fig. 2] Except at the lowest temperatures, an increased number of water molecules in the solvation shell of non-polar solute particles increases the repulsion between solute and solvent, resulting in a reduction of solubility, in an enhanced effective attraction between solute particles due to the decreased interface with water as they approach each other, and thus in aggregate stabilisation.

Monte Carlo simulations confirm the expectation that the cosolvent concentration is lower in shell water than in bulk water. Figure 7 shows the cosolvent shell concentration compared to the overall cosolvent concentration in the solution, where preferential exclusion of the kosmotropic cosolvent from the solvation shell of the hydrophobic solute particles is observed. At constant chem- 


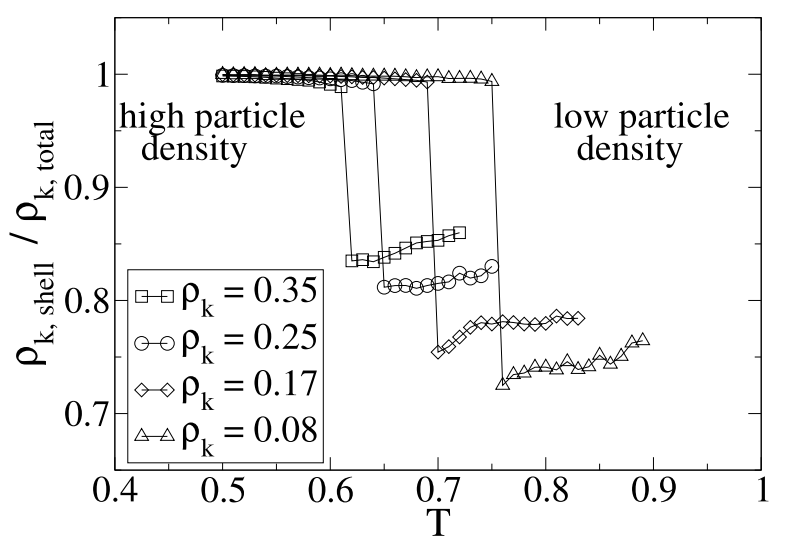

FIG. 7: Relative concentration of kosmotropic cosolvent in the solvation shell of hydrophobic particles, obtained from Monte Carlo simulations at chemical potential $\mu=2.5$, exhibiting preferential exclusion. The effect is most pronounced for low cosolvent densities.

ical potential $\mu$, a sharp drop occurs at the phase transition temperature (Fig. 6), which is due to the sudden change in solute particle density. At very low particle density, a clear exclusion of the cosolvent from the solvation shell appears. However, at high particle density (for temperatures below the aggregation phase transition) the effect is only marginal, because here the number of particle sites becomes substantial, most solvent and cosolvent sites are shell sites and thus the total cosolvent density is almost identical to the shell cosolvent density. The comparatively strong fluctuations in relative cosolvent shell concentration at temperatures above the phase transition may be attributed to the very low particle density in the system. Small, thermal fluctuations in the number of shell sites occupied by cosolvent molecules result in large fluctuations in the cosolvent shell concentration.

Considering next the effect of the cosolvent concentration, the system shows a substantially stronger preferential exclusion for low than for high cosolvent densities. This is again largely a consequence of considering the relative, as opposed to absolute, decrease in cosolvent concentration in the shell. Preferential exclusion is less pronounced for high cosolvent densities because a smaller proportion of cosolvent sites can contribute to the effect. At high temperatures, entropy, and therefore mixing, effects become dominant and the preferential exclusion of cosolvent particles shows a slight decrease.

We conclude this section by commenting also on the phenomenon shown in Figs. 4 and 5 that for temperatures where no aggregation is possible in the pure watersolute solution (below the LCST), it can be induced only by the addition of a cosolvent. Aggregation is driven by exclusion of solute from the solution in this intermediate regime, and is thus an indirect result of the transfor-

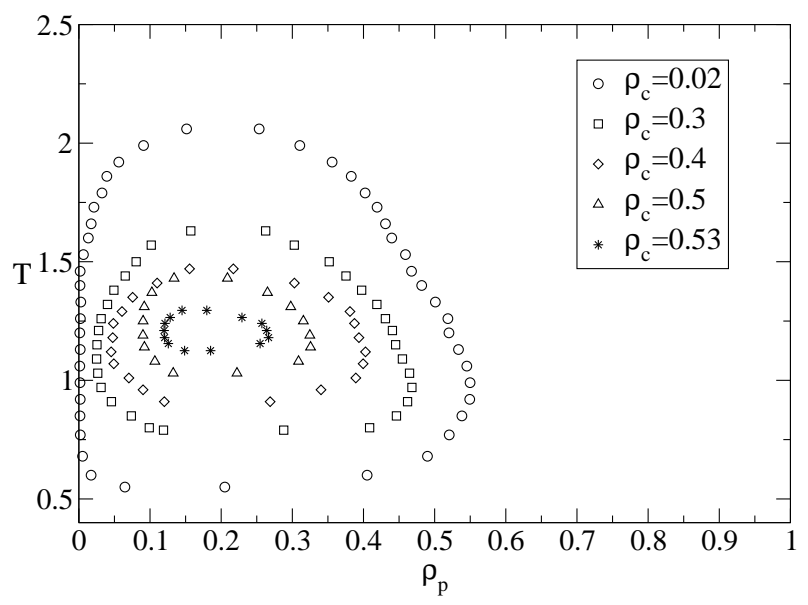

FIG. 8: Closed-loop miscibility curves of an aqueous solution of hydrophobic particles for different chaotropic cosolvent concentrations $\rho_{c}$, obtained by Monte Carlo simulations using the degeneracy parameters $\eta_{b}=9.0$ and $\eta_{s}=0.1$ in eq. (2). On the outside of the curves is the homogeneous, dissolved state while inside them is the aggregated state. As the cosolvent concentration grows, particle solubility increases, leading to a reduced coexistence regime.

mation of disordered bulk water states into energetically favourable ordered bulk states by the kosmotropic cosolvent. Preferential hydration is a further manifestation of this exclusion.

\section{CHAOTROPIC EFFECT}

A detailed study of chaotropic cosolvent effects within the adapted MLG model for a hydrophobic-polar mixture was presented in Ref. [36]. Here we provide only a brief review of the results of this analysis, with emphasis on the differences and similarities in comparison with the kosmotropic effect, which we have discussed at greater length in Sec. III] Figure 8 shows the coexistence curves demarcating the aggregated state for a range of cosolvent concentrations, computed by Monte Carlo simulations. The general features of the phase diagram are those of Sec. III)(Figs. 4land 5), with the obvious exception of the shrinking of the coexistence regime as cosolvent concentration is increased. However, it appears that for the $q$ and $\eta$ parameters used in the analysis (Sec. IIIB) the effect of high chaotropic cosolvent concentrations is rather stronger than that obtained with the kosmotropic cosolvent, an issue to which we return in Sec. V Figure 8 suggests that sufficiently high concentrations of chaotropic cosolvent may cause complete aggregate destabilisation at any temperature and solute particle density.

Figure 9 shows the corresponding $\mu-T$ phase diagram. As in Fig. [6] the lines representing the discontinuous jump in density from the low- to the high- $\rho_{p}$ phase move to higher $\mu$ with increasing cosolvent concentration, again reflecting the increased cost of adding water to the sys- 


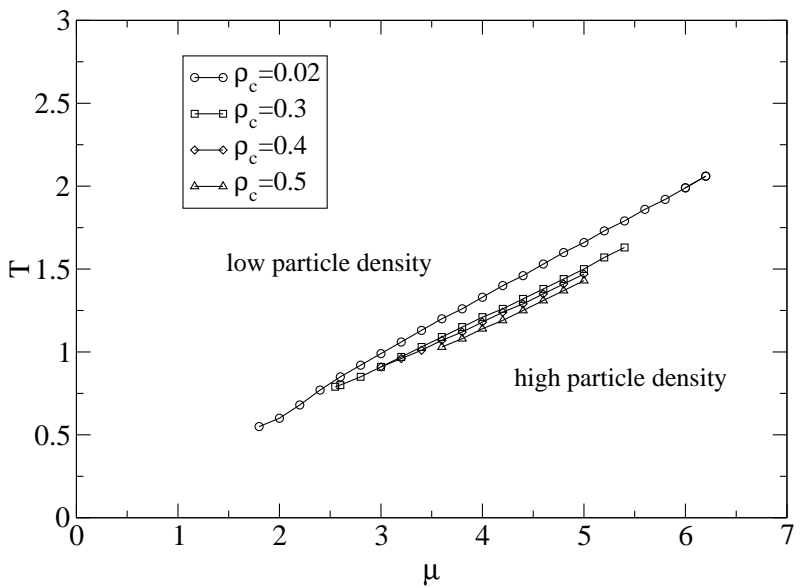

FIG. 9: $\mu$-T phase diagram of hydrophobic solute in an aqueous cosolvent mixture for different chaotropic cosolvent concentrations $\rho_{c}$, obtained by Monte Carlo simulation. The finite transition lines terminate at an UCST and a LCST, which approach each other with increasing cosolvent concentration.

tem in the presence of cosolvent. However, in this case the endpoints of the lines, which represent the UCST and LCST for each cosolvent concentration, become closer as $\rho_{c}$ increases until they approach a singularity (Fig. 8), which we discuss in detail in Sec. D

Figure 10] shows as a function of temperature the cosolvent concentration in the shell of a solute particle normalised by the total $\rho_{c}$. This demonstration of preferential binding of chaotropic agents to non-polar solute particles was obtained from the model of eq. (31) within a pair approximation, which was shown in Ref. [36] to provide a semi-quantitative reproduction of the Monte Carlo results. As in Fig. 7 the sharp step at the phase transition is due to the sudden change in particle density. At low particle densities (below the transition) the effect is clear, whereas at high densities it is small because the majority of solvent and cosolvent sites have become shell sites. For low cosolvent concentrations, preferential binding can lead to a strong increase in $\rho_{c}$ on the shell sites (80\% in Fig. 10), but the relative enhancement becomes less significant with increasing total cosolvent concentration. Finally, in counterpoint to the discussion at the end of Sec. III we comment that for chaotropic agents, which in bulk water transform ordered states into disordered ones, preferential binding (cosolvent exclusion) is favoured and solute exclusion decreases. Thus a regime exists in the $\rho_{p}-T$ phase diagram where at fixed temperature and particle density, aggregates of solute particles may be made to dissolve simply by the addition of cosolvent.

\section{STRONG AND WEAK COSOLVENTS}

As indicated in Sec. [II and illustrated by the results of Secs. III and IV there is a qualitative symmetry be-

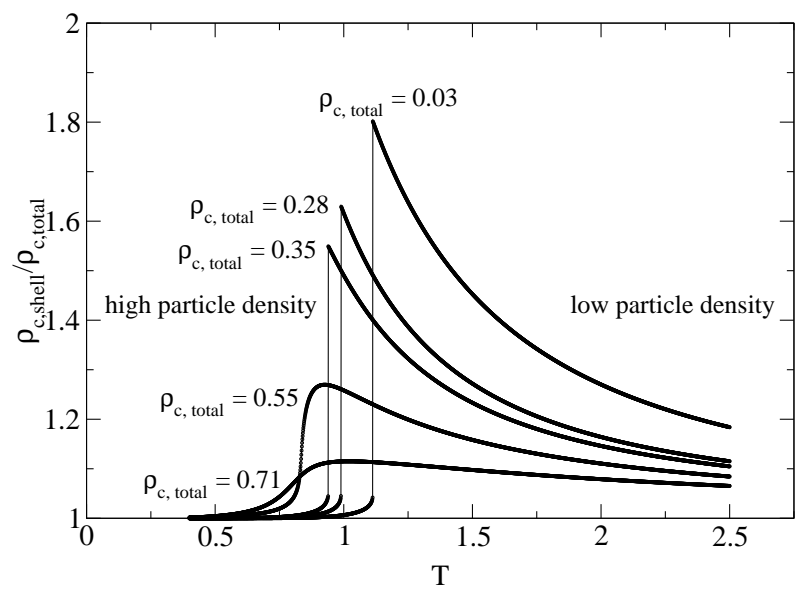

FIG. 10: Preferential binding effect, illustrated by the relative concentration of chaotropic cosolvent in the solvation shell of hydrophobic particles, for different cosolvent concentrations at fixed $\mu=3.0$, obtained by pair approximation.

tween the opposing effects of kosmotropic and chaotropic substances on hydrophobic aggregation of a given solute species. Indeed, the competition of the two effects has been studied for solutions of the protein HLL with the (denaturing) chaotrope guanidine hydrochloride and the (native-structure stabilising) kosmotrope betaine [8]. We remark, however, that the same cosolvent may have a different effect for solutions of different types and sizes of solute (Sec. II), and for this reason a global characterisation of the effectiveness of any one agent may be misleading.

At low cosolvent concentration, the stabilising or destabilising effects of the two cosolvent types on aggregate formation are rather small, and appear almost symmetrical in the expansion or contraction of the coexistence region (Figs. 5 and 8 Figs. 6] and 9). For weakly kosmotropic and chaotropic agents, by which is meant those causing only small enhancement or suppression of aggregation even at high concentrations, such qualitative symmetry might be expected as a general feature of the coexistence curves. With the same caveats concerning low concentrations or weak cosolvent activity, this statement is also true for preferential exclusion and binding of cosolvent to the solute particles, as shown by comparing Figs. [7and 10 However, differing phenomena emerge for strongly kosmotropic and chaotropic agents.

\section{A. Urea as a Strong Chaotrope}

Chaotropic cosolvents act to suppress the formation of water structure and, because the latter is already disrupted by the presence of a non-polar solute particle, are more efficient for bulk than for shell sites. Within the adapted MLG model, a maximally chaotropic agent is one which causes sufficient disruption that the number 

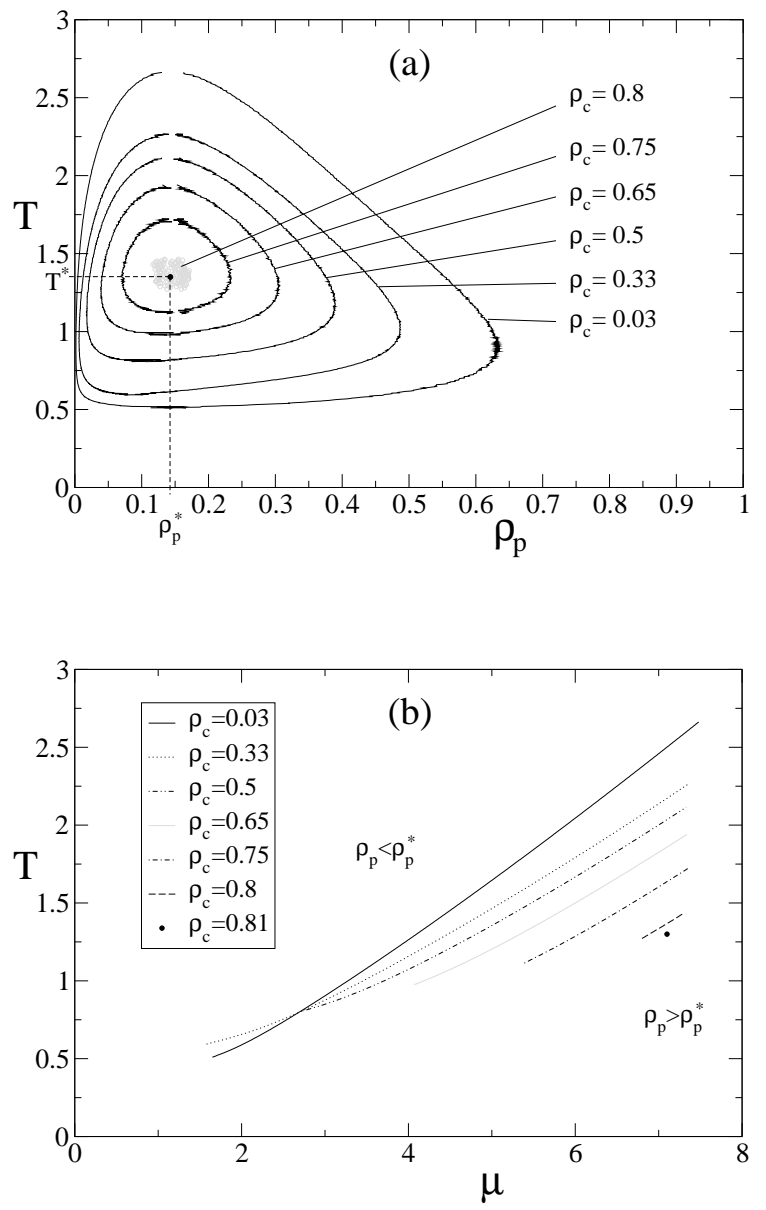

FIG. 11: (a) Coexistence curves for ternary water/cosolvent/solute systems with different cosolvent concentrations $\rho_{c}$, obtained by mean-field calculation. At a critical cosolvent concentration of $\rho_{c}^{*}=0.81$, the LCST and UCST coincide, at a critical temperature $T^{*}=1.35$ and critical particle density $\rho_{p}^{*}=0.14$, where the aggregation regime is reduced to a single point. For still higher cosolvent concentrations the solution is homogeneous over the full range of temperature and particle density. (b) Mean-field $\mu$ - $T$ phase diagram for different cosolvent concentrations. As $\rho_{c}$ increases, the separation of LCST and UCST decreases until for $\rho_{c}=\rho_{c}^{*}$ they meet at the temperature $T_{L}^{*}=T_{U}^{*}=T^{*}$, where the value $\mu_{L}=\mu_{U}=7.05$ determines $\rho_{p}^{*}=0.14$.

of ordered bulk states is reduced to its hypothetical minimum value, which is equal to the number of ordered shell states; by the definition based on hydrogen-bond formation, it is not possible to have fewer ordered bulk than ordered shell states, so $q_{o b} \geq q_{o s}$. When $q_{o b}$ and $q_{o s}$ become similar, the entropic gain of creating ordered bulk states by aggregation (removal of shell sites) is largely excluded, and thus a strongly chaotropic agent raises the solubility of hydrophobic particles very significantly. With a sufficient concentration of such cosolvents, aggregation may be completely prevented at all temperatures and densities, and the coexistence regime vanishes at a critical value of cosolvent concentration.
Such singular behaviour is well known in many aqueous solutions containing urea, and indeed frequent use is made of the destabilising properties of this cosolvent (Sec. I). We stress at this point that the chaotropic action of urea is by no means universal: in a number of systems, such as the ultra-small solute methane [20, 54], and proteins with variable hydrophobicity [49] or significant interactions between chain segments and the cosolvent molecules [52, 53], its effect may in fact be inverted. However, because of its ubiquity as a solubilising agent for most binary systems, we continue to consider urea as a generic example of a strong chaotrope.

The aggregation singularity was reproduced in the qualitative analysis of urea as a chaotropic agent performed in Ref. 36], and is illustrated in Fig. 11] Because of the discrete nature of the Monte Carlo simulations, the exact parameters for the critical point cannot be found using this technique; these were instead obtained only within the mean-field approximation, and as a result cannot be considered to be quantitatively accurate for the parameters of the system under consideration. Figure 11(a) shows the shrinking of the coexistence curves with increasing cosolvent concentration until they vanish at a critical value $\rho_{c}^{*}=0.81\left(c f . \rho_{c}^{*} \approx 0.55\right.$ expected from inspection of the Monte Carlo results in Fig. \&). The corresponding evolution of the transition line in the $\mu-T$ phase diagram is shown in Fig. 111(b).

With the canonical choice of degeneracy parameters (Sec. 【B) $q_{d s}=49, q_{d b}=40, q_{o b}=10$ and $q_{o s}=1$ [41], the values $\eta_{b}=9.0$ and $\eta_{s}=0.1$ required in eq. (2) to reproduce this critical vanishing are indeed such that $q_{o b, c}=1$ and $q_{o s, c}=0.9$ become very close. The level of "fine-tuning" of the underlying parameters required in the adapted MLG model reflects not a weakness of the framework but rather of the strongly chaotropic properties of urea in water: the destruction of hydrogen-bonded networks is almost complete at high concentrations. In practice, concentrated urea is used to destabilise aqueous solutions of many folded proteins, micelles and aggregates of hydrophobic particles. While we are unaware of systematic experimental studies of the effects of urea on the extent of the coexistence regime, aggregate destabilisation has been achieved at high concentrations in certain systems. At room temperature, the solubility of the highly hydrophobic amino-acid phenylalanin is doubled in an $8 \mathrm{M}$ urea solution (a solution with equal volume fractions of urea and water) [16].

\section{B. Sodium Chloride as a Weak Kosmotrope}

Kosmotropic cosolvents increase the extent of water structure formation, raising the number of ordered bulk and shell sites. While this process may be more efficient for ordered shell states, their degeneracy cannot exceed that of ordered bulk states. A maximally kosmotropic agent is one which causes sufficient structural enhancement that the number of ordered bulk states rises to the 


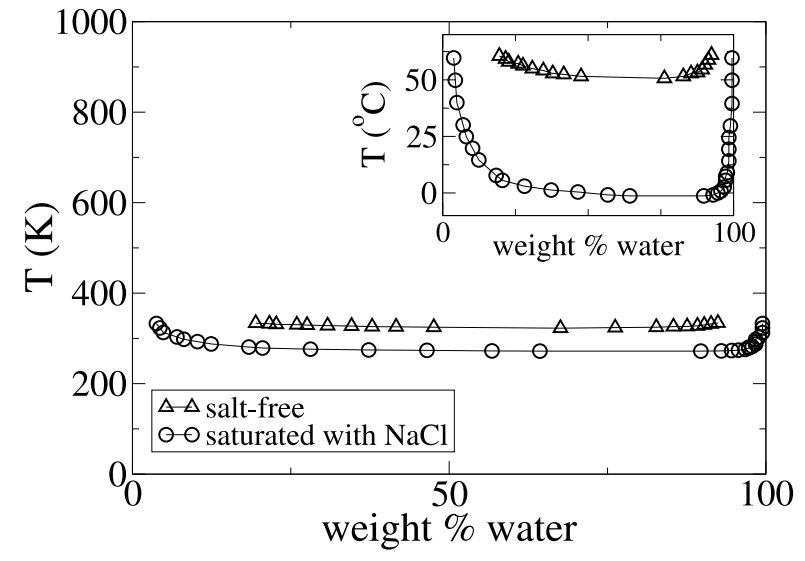

FIG. 12: Coexistence curve measured in the experimentally accessible temperature range for $\mathrm{N}, \mathrm{N}$-Diethylmethylamine in water in the presence and in the absence of the kosmotropic cosolvent sodium chloride, reproduced from Ref. 55]. The $\mathrm{LCST}$ is reduced from $51^{\circ} \mathrm{C}$ in the salt-free case to $-0.6^{\circ} \mathrm{C}$ in the saturated cosolvent solution.

point where it is equal to the number of disordered bulk states, $q_{o b}=q_{d b}$. Singular behaviour for a strongly kosmotropic cosolvent (the counterpart to the vanishing of aggregation caused by chaotropic cosolvents) is then the perfect aggregation of the system with densities $\rho_{1}=0$ and $\rho_{2}=1$ (Fig. 4), i.e. extension of the coexistence regime across the entire phase diagram. Only at the lowest temperatures, where entropy effects are irrelevant, would the mechanism of cage formation allow solution of the solute particles. For a sufficiently strong kosmotropic effect (high concentrations of a strong agent) there is no reason why $q_{o b}$ should not exceed $q_{d b}$, a situation which represents the breakdown of the sequence of energies and degeneracies characteristic of a system with a closed-loop miscibility curve (Sec. IIIA). Complete aggregation is the hallmark of this breakdown.

The "symmetrical" parameters $\eta_{b}=9.0$ and $\eta_{s}=0.1$ used in the analysis of Sec. III give the values $q_{o b}=19$ and $q_{d b}=31$ [eq. (10)]. These are clearly not sufficiently close to represent a strongly kosmotropic cosolvent, which would explain both the rather modest expansion of the coexistence regime even with relatively high cosolvent concentrations $\left(\rho_{k}\right)$ and the small values

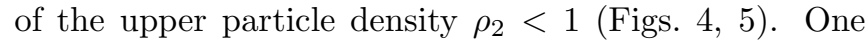
may thus conclude that quantitative symmetry between kosmotropic and chaotropic effects cannot be expected for any temperatures or densities, except in the event of very carefully chosen degeneracy parameters.

To our knowledge, rather little data is presently available concerning the phase diagram of hydrophobic solute particles in cosolvent solutions. However, Ting et al. 55. have measured the lower coexistence curves for N,NDiethylmethylamine both in pure water and in a mixture of water and the cosolvent sodium chloride (Fig. 12), which is usually considered to be weakly kosmotropic. A decrease of the LCST from $51^{\circ} \mathrm{C}$ to $-0.6^{\circ} \mathrm{C}$ is observed for a solution saturated with sodium chloride, corresponding to extension of the aggregation regime over a significantly greater temperature range. Although the data do not extend to sufficiently high temperatures to discuss the full extension of the aggregation regime in density (Fig. [12 inset), the qualitative similarity of Figs. 4 and 5 to the experimentally determined coexistence curves (Fig. 12 main panel) suggests that sodium chloride would be appropriately classified as a "weak" kosmotrope in its effect on N,N-Diethylmethylamine. While its activity is significantly stronger (or its saturation concentration significantly higher) than that of sodium sulphate, investigated by the same authors [55], at maximal concentrations it appears that the aggregation singularity is not reached and the closed-loop form of the miscibility curve is preserved. Here we note that, while the kosmotropic action of sodium chloride is due to its ionic nature in solution, the considerations of the adapted MLG framework (Sec. IB $)$ remain valid to model this effect. A more detailed comparison of the model results with the data is precluded by the fact that the cosolvent concentration in the saturated solution changes with both temperature and solute density. Measurements of different solutions are required to characterise the dependence of aggregation enhancement on the molecular properties of the solute and on both solute and cosolvent concentrations in the system.

Finally, preferential exclusion of kosmotropic cosolvents from the immediate vicinity of hydrophobic particles in aqueous solutions is measured only for very high cosolvent concentrations. Concentrations of compatible osmolytes, such as sucrose and betaine in the cytoplasm, typically reach values well in excess of $0.5 \mathrm{M}$. The stability of the protein lactate dehydrogenase against thermal denaturation increases by approximately $90 \%$ in a $1 \mathrm{M}$ sucrose solution at room temperature, and by $100 \%$ for the same concentration of hydroxyecotine [1], classifying these cosolvents as being strongly kosmotropic. In the current model it is difficult to specify the exact cosolvent concentration because one site contains a group of water molecules, but the results are nevertheless qualitatively consistent with observation. We have found that, except for specifically chosen values of the parameters $q_{\sigma}$ and $\eta_{\sigma}$, cosolvent concentrations well in excess of $10 \%$ are required to show a clear stabilising effect on hydrophobic aggregates (Fig. 5).

\section{SUMMARY}

We have discussed the physical mechanism underlying the action of kosmotropic and chaotropic cosolvents on hydrophobic aggregates in aqueous solutions. The aggregation phenomena arise from a balance between enthalpic and entropic contributions to the solvent free energy, which is fully contained within an adapted version of the 
bimodal model of Muller, Lee and Graziano. This model considers two populations of water molecules with differing physical properties, and generates indirectly the effective hydrophobic interaction between solute particles. Such a description, based on the microscopic details of water structure formation around a solute particle, is expected to be valid for small solute species, characterised by lengthscales below $1 \mathrm{~nm}$, and for those larger solute particles whose solubility is dominated by the atomically rough (highly curved) nature of their surfaces.

In the adapted MLG framework, one may include the ability of kosmotropic cosolvents to enhance and of chaotropic cosolvents to disrupt the structure of liquid water simply by altering the state degeneracies [eqs. (11) and (2)] associated with the two populations characterising this structure. As a consequence only of these changes in the properties of the solvent, the model reproduces all of the physical consequences of cosolvents in solution: for kosmotropes the stabilisation of aggregates, expansion of the coexistence regime and preferential cosolvent exclusion from the hydration shell of hydrophobic solute particles; for chaotropes the destabilisation of aggregates, a contraction of the coexistence regime and preferential binding to solute particles.

The microscopic origin of these preferential effects lies in the energetically favourable enhancement of hydrogenbonded water structure in the presence of a kosmotropic cosolvent, and conversely the avoidance of its disruption in the presence of a chaotrope. In a more macroscopic interpretation, the preferential hydration of solute particles by kosmotropic agents can be considered to strengthen the solvent-induced, effective hydrophobic interaction be- tween solute particles, thus stabilising their aggregates; similarly, the preferential binding of chaotropic agents to solute particles reduces the hydrophobic interaction, causing the particles to remain in solution. The essential cosolvent phenomena may thus be explained purely by the propensity of these agents to promote or suppress water structure formation, and the effectiveness of a cosolvent is contained in a transparent way in the microscopic degeneracy parameters of the solvent states.

Our focus on water structure is intended to extract one of the primary factors determining aggregation and cosolvent activity, and is not meant to imply that other interactions are not important. The range of solute species and additional contributions which are beyond the scope of our analysis includes extremely small solutes, large, atomically flat solute surfaces, solutes with differing surface hydrophobicity (such as polar side-groups) and surfaces undergoing specific chemical (or other) interactions with the cosolvent. However, from the qualitative trends, and even certain quantitative details, observed in a wide variety of aqueous systems it appears that the adapted MLG model is capable of capturing the essential phenomenology of cosolvent activity on aggregation and solubility for solutes as diverse as short hydrocarbons and large proteins.

\section{Acknowledgments}

We thank the Swiss National Science Foundation for financial support through grants FNRS 21-61397.00 and 2000-67886.02.
[1] E.A. Galinski, Compatible solutes of halophilic eubacteria: molecular principles, water-solute interaction, stress protection, Experientia 49 (1993) 487-496.

[2] P.H. Yancey, M.E. Clark, S.C. Hand, R.D. Bowlus and G.N. Somero, Living with water stress: evolution of osmolyte systems, Science 217 (1982) 1214-1222.

[3] G.N. Somero, Protons, osmolytes, and fitness of internal milieu for protein function, Am. J. Physiol. 251 (1986) R197-R213.

[4] P.M. Wiggins, Role of water in some biological processes, Microbiol. Rev. 54 (1990) 432-449.

[5] E. A. Galinski, M. Stein, B. Amendt and M. Kinder, The kosmotropic (structure-forming) effect of compensatory solutes, Comp. Biochem. Physiol. 117A (1997) 357-365.

[6] T. Arakawa and S.N. Timasheff, Mechanism of poly(ethylene glycol) interaction with proteins, Biochemistry 24 (1985) 6756-6762.

[7] S.N. Timasheff, in: Water and life, ed. G.S. Somero (Springer Verlag, Berlin, 1992) p. 70.

[8] T. Söderlund, K. Zhu, A. Jutila and P.K.J. Kinnunen, Effects of betaine on the structural dynamics of Thermomyces (Humicola) lanuginosa lipase, Coll. Surf. B: Biointerfaces 26 (2003) 75-83.

[9] D.W. Bolen and I.V. Baskakov, The osmophobic effect: natural selection of a thermodynamic force in protein folding, J. Mol. Biol. 310 (2001), 955-963.

[10] J.A. Schellman, Selective binding and solvent denaturation, Biopolymers 26 (1987) 549-559; A simple model for solvation in mixed solvents, Biophys. Chem. 37 (1990) 121-140; The relation between the free energy of interaction and binding, Biophys. Chem. 45 (1993) 273-279.

[11] S.N. Timasheff, Thermodynamic binding and site occupancy in the light of the Schellman exchange concept, Biophys. Chem. 101-102 (2002) 99-111.

[12] A. Tovchigrechko, M. Rodnikova and J. Barthel, Comparative study of urea and tetramethylurea in water by molecular dynamics simulations, J. Mol. Liq. 79 (1999) 187-201.

[13] J.R. De Xammar Oro, Role of co-solute in biomolecular stability: glucose, urea and the water structure, J. Biol. Phys. 27 (2001) 73-79.

[14] Y. Feng, Z.-W. Yu and P.J. Quinn, Effect of urea, dimethylurea, and tetramethylurea on the phase behavior of dioleoylphosphatidylethanolamine, Chem. Phys. Lipids 114 (2002) 149-157.

[15] T.H. Plumridge and R.D. Waigh, Water structure theory and some implications for drug design, J. Pharm. Pharmacol. 54 (2002) 1155-1179. 
[16] Y. Nozaki and C. Tanford, The solubility of amino acids and related compounds in aqueous urea solutions, J. Biol. Chem. 238 (1963) 4074-4081.

[17] Y. Nozaki and C. Tanford, The solubility of amino acids, diglycine, and triglycine in aqueous guanidine hydrochloride solutions, J. Biol. Chem. 245 (1970) 1648-1652.

[18] P.K. Nandi and D.R. Robinson, Effects of urea and guanidine hydrochloride on peptide and non-polar groups, Biochemistry 23 (1984) 6661-6668.

[19] G.E. Walrafen, Raman spectral studies of the effects of urea and sucrose on water structure, J. Chem. Phys. 44 (1966) 3726-3727.

[20] D.B. Wetlaufer, S.K. Malik, L. Stoller and R.L. Coffin, Nonpolar group participation in the denaturation of proteins by urea and guanidinium salts, J. Am. Chem. Soc. 86 (1967) 508-514.

[21] M. Roseman and W.P. Jencks, Interactions of urea and other polar compounds in water, J. Am Chem. Soc. 97 (1975) 631-640.

[22] Y. Kita, T. Arakawa, T.-Y. Lin and S.N. Timasheff, Contribution of the surface free energy perturbation to protein-solvent interactions, Biochemistry 33 (1994) 15178-15189.

[23] F. Franks, Protein stability: the value of 'old literature', Biophys. Chem. 96 (2002) 117-127, and references therein.

[24] S.N. Timasheff and T. Arakawa, in: Protein structure: a practical approach, ed. T.E. Creighton (IRL Press, Oxford, 1997) p. 331.

[25] H.S. Frank and M.W. Evans, Free volume and entropy in condensed systems, J. Chem. Phys. 13 (1945) 507-532.

[26] M. Yaacobi and A. Ben-Naim, Solvophobic interaction, J. Phys. Chem. 78 (1974) 175-178.

[27] P.L. Privalov and S.J. Gill, Stability of protein structure and hydrophobic interaction, Adv. Protein Chem. 39 (1988) 191-234.

[28] P.H.K. De Jong, J.E. Wilson and G.W. Neilson, Hydrophobic hydration of methane, Mol. Phys. 91 (1997) 99-103.

[29] A. Perstemilidis, A.M. Saxena, A.K. Soper, T. HeadGordon and R.M. Glaeser, Direct evidence for modified solvent structure within the hydration shell of a hydrophobic amino acid, Proc. Natl. Acad. Sci. USA 93 (1996) 10769-10774.

[30] W. Kauzmann, Some factors in the interpretation of protein denaturation, Adv. Protein Chem. 14 (1959) 1-63.

[31] C. Tanford, The hydrophobic effect: formation of micelles and biological membranes (Wiley, New York, 1980).

[32] F.H. Stillinger, Water revisited, Science 209 (1980) 451457.

[33] A. Ben-Naim, Water and aqueous solutions: introduction to a molecular theory (Plenum Press, New York, 1974).

[34] R. Ludwig, Water: from clusters to the bulk, Angew. Chem. Int. Ed. Engl. 40 (2001) 1808-1827.

[35] S.N. Timasheff, Protein-solvent preferential interactions, protein hydration, and the modulation of biochemical reactions by solvent components, Proc. Natl. Acad. Sci. 99 (2002) 9721-9726.

[36] S. Moelbert and P. De Los Rios, Chaotropic effect and preferential binding in a hydrophobic interaction model, J. Chem. Phys. 119 (2003) 7988-8001.

[37] N. Muller, Search for a realistic view of hydrophobic effects, Acc. Chem. Res. 23 (1990) 23-28.
[38] B. Lee and G. Graziano, A two-state model of hydrophobic hydration that produces compensating enthalpy and entropy changes, J. Am. Chem. Soc. 22 (1996) 5163-5168.

[39] K.A.T. Silverstein, A.D.J. Haymet and K.A. Dill, The strength of hydrogen bonds in liquid water and around non-polar solute, J. Am. Chem. Soc. 122 (2000) 80378041.

[40] S. Moelbert and P. De Los Rios, Hydrophobic interaction model for upper and lower critical solution temperatures, Macromolecules 36 (2003) 5845-5853.

[41] K.A.T. Silverstein, A.D.J. Haymet and K.A. Dill, Molecular model of hydrophobic solvation, J. Chem. Phys. 111 (1999) 8000-8009.

[42] D. Paschek, Temperature dependence of the hydrophobic hydration and interaction of simple solutes: an examination of five popular water models, J. Chem. Phys. 120 (2004) 6674-6690; Heat capacity effects associated with the hydrophobic hydration and interaction of simple solutes: a detailed structural and energetical analysis based on molecular dynamics simulations, J. Chem. Phys. 120 (2004) 10605-10617.

[43] P. De Los Rios and G. Caldarelli, Cold and warm swelling of hydrophobic polymers, Phys. Rev. E 63 (2001) 0318021-5.

[44] P. De Los Rios and G. Caldarelli, Putting proteins back into water, Phys. Rev. E 62 (2000) 8449-8452.

[45] G. Caldarelli and P. De Los Rios, Cold and warm denaturation of proteins, J. Biol. Phys. 27 (2001) 229-241.

[46] S. Moelbert, B. Normand and P. De Los Rios, Solventinduced micelle formation in a hydrophobic interaction model, Phys. Rev. E 69 (2004) 061924-1-11.

[47] C.Y. Lee, J.A. McCammon and P.J. Rossky, The structure of liquid water at an extended hydrophobic surface, J. Chem. Phys. 80 (1984) 4448-4453.

[48] X. Huang, C.J. Margulis and B.J. Berne, Do molecules as small as neopentane induce a hydrophobic response similar to that of large hydrophobic surfaces ? J. Phys. Chem. B 107 (2003) 11742-11748.

[49] S. Shimizu and H.S. Chan, Origins of protein denatured states compactness and hydrophobic clustering in aqueous urea: inferences from nonpolar potentials of mean force, Proteins: Struct. Funct. Genet. 49 (2002) 560-566.

[50] K. Lum, D. Chandler and J.D. Weeks, Hydrophobicity at small and large length scales, J. Phys. Chem. 103 (1999), 4570-4577.

[51] E.G. Finer, F. Franks and M.J. Tait, Nuclear magnetic resonance studies of aqueous urea solutions, J. Am. Chem. Soc. 94 (1972) 4424-4427.

[52] J.M. Scholtz, D. Barrick, E.J. York, J.M. Stewart and R.L. Baldwin, Urea unfolding of peptide helices as a model for interpreting protein unfolding, Proc. Natl. Acad. Sci. USA 92 (1995) 185-189.

[53] R.A. Kuharski and P.J. Rossky, Solvation of hydrophobic species in aqueous urea solution: a molecular dynamics study, J. Am. Chem. Soc. 106 (1984) 5794-5800.

[54] G. Graziano, On the solubility of aliphatic hydrocarbons in $7 \mathrm{M}$ aqueous urea, J. Phys. Chem. B 105 (2001) 26322637.

[55] A.M. Ting, S. Lynn and J.M. Prausnitz, Liquidliquid equilibria for aqueous systems containing N,NDiethylmethylamine and sodium chloride or sodium sulfate, J. Chem. Eng. Data 37 (1992) 252-259. 